\title{
Cross-modality priming in stem completion reflects conscious memory, but not voluntary memory
}

\author{
ALAN RICHARDSON-KLAVEHN \\ University of Westminster, London, England \\ and \\ JOHN M. GARDINER \\ City University, London, England
}

\begin{abstract}
A comparison of incidental and intentional stem-completion tests confirmed that cross-modality priming occurs when performance conforms completely to the retrieval intentionality criterion, indicating involuntary-not voluntary-retrieval in the incidental test. However, an on-line measure of awareness in the incidental test, and a process-dissociation analysis of the intentional test, indicated only within-modality, but not cross-modality, transfer of involuntary retrieval that is unaccompanied by memorial awareness. These results imply that conscious memory should not be equated with voluntary retrieval, and unconscious memory should not be equated with involuntary retrieval, because involuntary retrieval can be accompanied by memorial awareness.
\end{abstract}

Incidental perceptual tests of memory typically require participants to complete or identify an incomplete or degraded external stimulus (e.g., a word stem or a briefly flashed word). Prior study of the intact version of a test item facilitates or "primes" current performance. This priming is characterized as perceptual because it often varies with the degree of perceptual match between study and test items: In a visual test, for example, priming decreases markedly when study is auditory compared to when it is visual (for reviews, see Richardson-Klavehn \& Bjork, 1988; Roediger \& McDermott, 1993). Such findings, among others, have led to the hypothesis that priming depends on modality-specific perceptual representation systems (see, e.g., Tulving \& Schacter, 1990). These systems are subserved by brain areas involved in perceptual analysis (see, e.g., Schacter et al., 1995), and their operation is not amenable to voluntary control.

Cross-modality priming is weaker than within-modality priming, but significant cross-modality priming is still observed in a variety of tests (see, e.g., Rajaram \& Roediger, 1993; for a review, see Toth \& Reingold, 1996). Whereas there is strong evidence that within-modality priming can occur without voluntary retrieval of study list items (see, e.g., Richardson-Klavehn, Gardiner, \& Java, 1994; Richardson-Klavehn, Lee, Joubran, \& Bjork,

\footnotetext{
We are most grateful to Rina Kakad for testing the participants, and to Eyal Reingold, Roddy Roediger, and two anonymous reviewers for constructive comments on an earlier version of this paper. Preparation of this paper was facilitated by Grant R000-23-6225 from the Economic and Social Research Council (U.K.). Correspondence should be addressed to A. Richardson-Klavehn, Division of Psychology, University of Westminster, 309 Regent St., London W1R 8AL, England (email: a.r.klavehn@wmin.ac.uk).
}

1994), the situation with respect to cross-modality priming is unclear. It could reflect "contamination" by voluntary retrieval, because voluntary retrieval typically depends on modality-independent (particularly semantic) information. On the other hand, cross-modality priming could be involuntary, reflecting lexical representations (see, e.g., Weldon, 1991, 1993) or representations for verbal responses that accumulate information across input modalities (see, e.g., Kirsner, Dunn, \& Standen, 1989). Resolution of the issue of voluntary contamination is, therefore, critical to progress in understanding priming in incidental perceptual tests. Here we focus on crossmodality priming in the word-stem completion task, and we contrast two methods of detecting voluntary contamination, the process-dissociation procedure (Jacoby, 1991) and the retrieval intentionality criterion (Schacter, Bowers, \& Booker, 1989). We show that these methods lead to divergent conclusions about whether cross-modality priming reflects contamination and provide a resolution of the apparent discrepancy.

In the process-dissociation procedure, it is assumed that controlled (voluntary) and automatic (involuntary) retrieval are independent, and a comparison of inclusion and exclusion tests is prescribed. In stem completion, the inclusion test involves attempting to complete each stem with a studied word, but if a studied word cannot be retrieved, completing the stem with the first word that comes to mind. The exclusion test involves completing each stem, but not with a studied word. Controlled $(C)$ and automatic $(A)$ retrieval are assumed to act in concert in the inclusion test, so that the probability of completing a stem with a studied word is equal to $C+A(1-C)$. In the exclusion test, a studied completion will be given only when automatic retrieval is not overridden by controlled 
retrieval, so that the probability of a studied completion is equal to $A(1-C)$. The difference in performance between the tests is treated as an estimate of the probability of controlled retrieval $(C)$. Under the independence assumption, the probability of automatic retrieval is then estimated by dividing exclusion test performance by $(1-C)$. This operation boosts exclusion performance to account for trials on which controlled and automatic retrieval jointly operate [i.e., $A=E X C /(1-C)=E X C+$ $(C A)]$. Automatic memory is said to be evident when the $A$ parameter exceeds baseline (the probability of completing a stem with a target word when that word has not been studied). Jacoby and colleagues (e.g., Jacoby, Toth, \& Yonelinas, 1993; Jacoby, Yonelinas, \& Jennings, in press) applied this procedure in experiments involving visual and auditory study conditions and visual stemand fragment-completion tests and found little evidence for cross-modality transfer of automatic retrieval. They (see also Toth \& Reingold, 1996) have argued that crossmodality priming in incidental tests reflects contamination by controlled retrieval.

In contrast to the process-dissociation procedure, the retrieval intentionality criterion relies on a traditional comparison of intentional and incidental tests, stipulating that the tests present identical retrieval cues (e.g., word stems), and that only the instructions vary. When a manipulation influences the tests differently, that dissociation can be attributed only to a difference in retrieval volition. Depth of processing at study is a popular manipulation. When participants are instructed to complete test items with studied words (intentional test), a strong advantage of deep over shallow processing usually occurs. By contrast, when participants are instructed to complete test items with the first word coming to mind (incidental test), priming should be uninfluenced by depth-of-processing, indicating that retrieval is involuntary (see, e.g., Roediger, Weldon, Stadler, \& Riegler, 1992). If priming is contaminated by voluntary retrieval, however, it should show a depthof-processing effect (see, e.g., Challis \& Brodbeck, 1992). The retrieval intentionality criterion has been criticized by some proponents of the process-dissociation procedure because it does not equate "response bias" across tests (e.g., Reingold \& Toth, 1996; Toth, Reingold, \& Jacoby, 1994). Response bias is assumed to vary when incidentaltest participants are required to complete every test cue, and intentional-test participants are not. However, patterns of data that satisfy the criterion are obtained even when the intentional test requires a response to every test cue (Richardson-Klavehn \& Gardiner, 1995).

Craik, Moscovitch, and McDowd (1994) applied the criterion to cross-modality priming in stem completion, using a visual test and visual and auditory study conditions. They found a strong advantage of deep over shallow processing in cross-modality stem-cued recall. By contrast, cross-modality priming showed no depth-ofprocessing effect at all, indicating involuntary retrievaland not voluntary contamination. In conjunction with the results of the process-dissociation procedure, these re- sults present a paradox. This paradox is particularly compelling because proponents of the procedure assert that the estimates of automatic retrieval it produces converge with uncontaminated measures of priming in incidental tests (e.g., Jacoby et al., in press; Toth, Reingold, \& Jacoby, 1995). In particular, it is agreed that the absence of a depth-of-processing effect in an incidental perceptual test indicates freedom from contamination (Reingold \& Toth, 1996; Toth \& Reingold, 1996; Toth et al., 1994).

We argue here that the paradox arises only if conscious awareness of memory is equated with voluntary retrieval of previously encountered information, as is done in the process-dissociation framework. Our alternative framework (Richardson-Klavehn, Gardiner, \& Java, 1996; see also Ebbinghaus, 1885/1964; Schacter, 1987) distinguishes memorial state of awareness (conscious vs. unconscious) from retrieval volition (voluntary vs. involuntary), so that involuntary retrieval can be accompanied by memorial awareness (involuntary conscious memory). In terms of our framework, "contamination" could mean that incidental-test participants deliberately respond with studied items, contrary to instructions. Or it could mean that they become aware that particular responses are studied items, even though their performance reflects involuntary retrieval. In consequence, even if voluntary retrieval is completely absent, incidental test performance reflects a mix of involuntary retrieval that is accompanied by conscious awareness of memory and involuntary retrieval that is unaccompanied by conscious awareness of memory.

The paradox would be resolved, therefore, if withinmodality priming in an incidental test reflects involuntary memory, both conscious and unconscious, but crossmodality priming reflects only involuntary conscious memory. This analysis implies that cross-modality priming does not reflect voluntary contamination, but that it will nevertheless fail to be reflected in the automatic retrieval parameter of the process-dissociation procedure. Items associated with involuntary conscious memory in an incidental test would be suppressed in an exclusion test because the exclusion instructions require suppression of items associated with awareness of occurrence in the study list. This suppression would occur whether studied items were voluntarily retrieved and associated with conscious awareness of memory, or involuntarily retrieved and associated with conscious awareness of memory. In terms of our framework, therefore, the estimate of automatic retrieval obtained from the process-dissociation procedure does not index involuntary memory, both conscious and unconscious, but only involuntary unconscious memory. The process-dissociation procedure does not distinguish voluntary conscious from involuntary conscious memory, so any priming in an incidental test that is not accompanied by transfer in the automatic (involuntary) retrieval parameter is attributed to controlled (voluntary) retrieval. The result would be the false conclusion that cross-modality transfer in an incidental test reflects contamination by voluntary retrieval of studied items. 
To test this hypothesis, we applied an empirical method developed by Richardson-Klavehn and Gardiner (1995) for analyzing retrieval volition and memorial awareness in stem completion. It involves incidental and intentional tests, and therefore applies the logic of the retrieval intentionality criterion. However, it deviates from previous methods in three ways: (1) There is an on-line recognition measure in the incidental test, so that participants indicate (by providing a second completion) when they are aware that their first completion was studied. (2) Participants in the intentional test attempt to complete each stem with a studied word. If successful, they provide a second completion to indicate that their first completion was recalled. If unsuccessful, they complete the stem with the first word that comes to mind. (3) Time to complete both tests is measured, providing convergent evidence concerning retrieval strategy.

The on-line recognition measure in the incidental test permits a measure of the extent to which priming is accompanied by recognition of prior occurrence. RichardsonKlavehn and Gardiner (1995) found that depth of processing had little influence on priming in this task, but it had a strong influence on recognition of completions as studied. Consistent with the distinction between retrieval volition and memorial awareness in our framework, this result demonstrates that conscious awareness of memory can vary dramatically, even when stem-completion priming reflects involuntary retrieval. Richardson-Klavehn and Gardiner (1995) also showed that the stem-completion rate for unrecognized items in this test accurately predicts performance in a variant of an exclusion test in which participants give the first completion that comes to mind, but omit and replace studied words. For that reason, we did not include this kind of test, termed an opposition test by Richardson-Klavehn, Gardiner, and Java (1994).

The intentional test in our procedure corresponds to an inclusion test in the process-dissociation procedure, and has two functions. First, it allows one to determine whether incidental-test performance conforms to the retrieval intentionality criterion, because stem-completion performance in the intentional test shows a strong depth-ofprocessing effect (Richardson-Klavehn \& Gardiner, 1995). Second, it permits a process-dissociation analysis to be performed on a single test, because it is possible to determine which words are recalled and which are given as the first word that comes to mind. It is reasonable to assume that recalled words would be suppressed and replaced in an exclusion test, so exclusion performance can be estimated by subtracting the proportion of stems completed with recalled words from the overall proportion of stems completed with target words. Participants are required to write an additional completion to indicate recall in order to equate all aspects of cognitive activity (e.g., lexical search) between the current test and a standard exclusion test. The logic is that the additional completions would replace the recalled completions in an exclusion test.
We prefer this revised method to the typical method involving separate inclusion and exclusion trials (see, e.g., Jacoby et al., 1993), because the separate-trials method permits different retrieval strategies on the different trials. Such strategic changes can violate the assumption that voluntary retrieval brings studied words to mind in response to the word-stem cues on both exclusion and inclusion trials. For example, the exclusion instructions used by Richardson-Klavehn, Gardiner, \& Java (1994) have been criticized by Jacoby and colleagues because they encourage involuntary-rather than voluntary-retrieval of studied words. And when voluntary retrieval is not recruited by the exclusion instructions, the independence model is held to be inapplicable (for discussion, see Jacoby, Begg, \& Toth, 1996; Reingold \& Toth, 1996; Richardson-Klavehn \& Gardiner, 1995; RichardsonKlavehn et al., 1996; Toth et al., 1995). The method used here not only allows one to verify that inclusion scores reflect voluntary retrieval of studied words (as evidenced by a depth-of-processing effect), but also ensures that exclusion scores reflect an equal (inhibitory) contribution of voluntary retrieval, because exclusion scores are derived from inclusion scores. Further advantages of our method are that it ensures that participants cannot confuse inclusion and exclusion trials, and that it permits a direct comparison with an incidental test, based on equal numbers of study and test items.

We combined the visual stem-completion tests with study manipulations of depth of processing and modality (visual vs. auditory). Our principal predictions were (1) that cross-modality priming would occur in the incidental test when performance conformed to the retrieval intentionality criterion, indicating involuntary retrieval (and replicating Craik et al., 1994); (2) that cross-modality priming would be associated with memorial awareness, as reflected in the on-line recognition measure in the incidental test, so that subtracting out recognized items would remove the cross-modality transfer evident in overall performance; and (3) that the automatic retrieval parameter obtained from the process-dissociation analysis would show no evidence of cross-modality transfer (consistent with the findings of Jacoby et al., 1993; Jacoby et al., in press).

\section{METHOD}

\section{Participants, Design, and Materials}

The participants were 32 undergraduate volunteers from an introductory psychology course at Middlesex University. In total, 35 participants were tested, with 3 ( 1 in the incidental test and 2 in the intentional test) being replaced because they failed to follow test instructions. The experiment was a $2 \times 2 \times 2$ mixed factorial design, with test instructions (incidental vs. intentional) as a between-group factor and study task [pleasantness rating (deep) vs. syllable counting (shallow)] and study modality (visual vs. auditory) as within-group factors. Assignment to test conditions was random, with 16 participants in each group. A separate study list was presented for each of the four study conditions, and the order of the study conditions was counterbalanced using a $4 \times 4$ Latin square. Each study list consisted of 20 words ( 16 critical words and 4 filler words). The test list consisted of 128 threeletter stems, with half of the stems corresponding to the 64 critical 
words presented at study and half corresponding to unstudied words. The studied/unstudied status of the test items was also counterbalanced, yielding eight unique study/test formats. Two participants in each test group were randomly assigned to each format.

The materials consisted of 128 critical words and 16 filler words. Of the critical words, 112 were selected from a pool of five-letter words suplied by Jacoby (personal communication), and 16 were selected from the six-letter words used by Richardson-Klavehn, Gardiner, and Java (1994). The critical words were either mono- or disyllabic and did not include homophones. Stems corresponding to these words were unique in the set of 128, and could be completed with at least two different English words. The 16 filler words were selected from the Concise Oxford English Dictionary and were tri- or quadrisyllabic. None could be used to complete stems corresponding to critical words. The critical words were assigned to eight sets of 16 words, so that the mean baserates for the sets were similar. With the addition of four filler words, these sets formed the eight 20 -word study lists (Lists A-H). Half the participants in each test group received Lists A, B, C, and D at study, and the other half received Lists E, F, G, and $H$. The lists that were studied were presented in a fixed order (e.g., A first, B second, C third, and D last), ensuring that each list appeared in each study condition (because the order of the study conditions was counterbalanced). The filler words were included in each study list so that syllable judgments ranged between 1 and 4 . The first and last items in each study list were fillers, and the remaining two fillers were inserted unsystematically among the critical items. Because each participant was exposed to only four of the eight study lists, Lists A and E shared fillers, as did Lists B and F, C and G, and $\mathrm{D}$ and $\mathrm{H}$. The test list consisted of stems corresponding to the critical items in Lists $\mathrm{A}-\mathrm{H}$, and was block randomized, with each eightword block containing one item from each list.

\section{Procedure}

The participants were tested individually and were told that the experiment involved simple verbal tasks. The four study lists were then presented, with appropriate instructions given prior to each list. The study lists were printed on cards in uppercase letters. In the visual conditions the experimenter showed these cards, whereas in the auditory conditions she read the words aloud from the cards. The participants judged either the pleasantness of the referent of each word $(1=$ least pleasant $; 4=$ most pleasant ) or the number of syllables in each word (1-4 scale). They recorded their judgments for each list on a sheet containing 20 numbered spaces. List presentation was paced by the participant, with the next word being presented after the judgment for a particular word was written. After the fourth study list, the participants completed a list of 34 stems with the first city coming to mind (e.g., $L O N$, $M O S)$. None of these stems corresponded to stems in the upcoming test. This distractor activity served to familiarize the participants with stemcompletion tasks and to accustom them to being timed by the experimenter. She activated an electronic stopwatch when they turned their attention to the first stem and turned it off when they completed the last stem.

The participants then completed a five-page test booklet. Each page of this booklet contained two columns of stems printed in uppercase letters, with the stems in the right-hand column being exact duplicates of the ones in the left-hand column. The incidental-test participants were told to work down the left column of each page, completing each stem with the first word coming to mind. They were told that words coming to mind might sometimes have been encountered in the first part of the experiment, owing to overlap in materials. When such a word came to mind, they were to write that word in the left-hand column, but they were also to complete the (duplicate) stem in the right-hand column with a different word, or to place a dash in the right-hand column if none came to mind. The experimenter strongly emphasized that it was not the purpose of the task to complete the stems with words encountered earlier. The intentional-test participants were told to work down the lefthand column of each page, attempting to complete each stem with a word from the study lists. If able to recall a studied word, they were to write that word in the left-hand column, but they were also to complete the (duplicate) stem in the right-hand column with a different word, or to place a dash in the right-hand column if none came to mind. If unable to recall a studied word, they were to complete the stem in the lefthand column with the first word that came to mind and to ignore the stem in the right-hand column. All participants were told that they should work through the test "quite briskly," and were timed, as in the distractor task. After the test, all participants were interviewed concerning their test strategy.

\section{RESULTS AND DISCUSSION}

The overall mean proportions of stems completed with target words are shown in Table 1, with the mean proportions of stems completed with recognized words (incidental test) and recalled words (intentional test) in parentheses. The results confirmed our predictions. First, the incidental test showed substantial cross-modality priming, but less cross-modality than within-modality priming. Second, depth of processing did not influence priming in the incidental test, but deep processing led to better overall performance than shallow processing in the intentional test. Third, this pattern occurred in the auditory study conditions, as well as the visual study conditions (replicating Craik et al., 1994). By the retrieval intentionality criterion, therefore, both cross- and within-modality priming in the incidental test reflected involuntary retrieval. Had cross-modality priming reflected contamination by voluntary retrieval, priming would have been influenced by depth of processing.

Inferential analyses supported these conclusions. A 2 $\times 2 \times 2$ mixed analysis of variance on the overall proportions of stems completed with studied targets revealed a significant interaction of test instructions and depth of processing at study $[F(1,30)=10.43, p<.005]$ and a significant main effect of study modality $[F(1,30)=$ $14.48, p<.001]$. No other effects were significant (all $F \mathrm{~s}<1.31$; all $p \mathrm{~s}>.25$ ), except for the main effect of depth of processing $[F(1,30)=10.07, p<.005]$, which was qualified by the significant interaction just described. Planned comparisons (averaged over study modality) revealed a nonsignificant effect of depth of processing in the incidental test $(F<1)$, but a highly significant effect in the intentional test $[F(1,30)=20.50, p<.001]$. Additional analyses compared each study condition with the unstudied baseline. Significant priming was observed in all four conditions in the incidental test $(t \mathrm{~s}=6.05,6.35,3.94$, and 3.87 for the visual-deep, visual-shallow, auditory-deep,

Table 1

Mean Proportions of Stems Completed With Targets, as a Function of Depth of Processing, Study Modality, and Test Instructions

\begin{tabular}{llll}
\hline & \multicolumn{2}{c}{ Studied } & \\
\cline { 2 - 3 } Study Modality & Deep & Shallow & Unstudied \\
Incidental Test & & \\
Visual & $.43(.34)$ & $.42(.07)$ & $.20(.00)$ \\
Auditory & $.32(.22)$ & $.33(.12)$ & \\
& Intentional Test & \\
Visual & $.47(.43)$ & $.36(.11)$ & $.21(.02)$ \\
Auditory & $.41(.33)$ & $.31(.11)$ & \\
\hline
\end{tabular}

Note-Mean proportions of stems completed with recognized words (incidental test) and recalled words (intentional test) are shown in parentheses. 
and auditory-shallow conditions, respectively; $d f=15$; all $p s<.001$ ). Performance in the intentional test was also significantly above baseline in all conditions $(t \mathrm{~s}=$ $5.99,5.11,7.40$, and $2.82 ; d f=15$; all $p s<.01$ ). Baseline performance did not differ between the tests $[t(30)=$ $-0.43, p>.5]$.

The completion-time data confirm that incidental-test performance reflected involuntary retrieval and replicate the findings of Richardson-Klavehn and Gardiner (1995). Each participant's total time to complete the test was divided by 128 to obtain the number of seconds per test item. The mean time per test item was $4.1 \sec (S D=0.9)$ for the incidental test and $6.8 \mathrm{sec}(S D=2.2)$ for the intentional test, a highly significant difference (MannWhitney, $U=162.0, p<.0005$ ). Voluntary retrieval, as engaged in the intentional test, was more effortful and time-consuming than was involuntary retrieval. ${ }^{1}$

On-line recognition of target completions as studied words in the incidental test was influenced by depth of processing for both visual and auditory items (sign tests, both $p s<.02$ ), despite the fact that priming in that test showed no depth-of-processing effect. This result replicates the pattern obtained by Richardson-Klavehn and Gardiner (1995) for items presented visually at study, but extends it to items presented auditorily at study. It demonstrates that awareness that completions are studied can vary dramatically as a function of depth of processing, even though overall stem-completion performance reflects involuntary retrieval. Table 2 shows the mean proportions of stems completed with unrecognized words. Performance in the visual-deep condition was below the unstudied baseline (sign test, $p<.005$ ). By contrast, significant facilitation occurred in the visual-shallow condition $[t(15)=4.04, p<.001]$. This pattern is exactly the same as the one obtained in an opposition test, in which participants complete stems with the first word that comes to mind, but omit and replace studied words (RichardsonKlavehn \& Gardiner, 1995; Richardson-Klavehn, Gardiner, \& Java, 1994). The critical new results come from the auditory conditions. Performance in the auditorydeep condition was below baseline, as was that in the vi-

Table 2

Mean Proportions of Stems Completed With Unrecognized Words and Mean $\boldsymbol{A}$ Estimates From the Process-Dissociation Analysis

\begin{tabular}{llll} 
& \multicolumn{2}{c}{ Studied } & \\
\cline { 2 - 3 } Study Modality & Deep & Shallow & Unstudied \\
\hline
\end{tabular}

Unrecognized Words (Incidental Test)

\begin{tabular}{lccc} 
Visual & .09 & .35 \\
Auditory & .10 & .21 & .20 \\
\multicolumn{4}{c}{ Automatic } \\
Visual & .07 & .27 & \\
Auditory & .12 & .22 &
\end{tabular}

Note- $A$ (automatic retrieval) was not computed for unstudied items. The mean baseline value in parentheses was obtained by averaging inclusion and exclusion values. sual-deep condition [sign test, $p<.025$ ]. However, in contrast to the visual-shallow condition, the auditory-shallow condition produced no facilitation for unrecognized words $[t(15)=0.12, p>.4]$. The results confirm the prediction that involuntary memory that is unaccompanied by conscious awareness of memory does not transfer across modalities.

The process-dissociation analysis was conducted on the data from the intentional test using the method outlined previously. The overall proportions of stems completed with studied words (means in Table 1) were the inclusion proportions, and the exclusion proportions were derived by subtracting the recall proportions (means in parentheses in Table 1) from the inclusion proportions. By implication, the recall proportions were the estimates of $C$ (controlled retrieval), because $C$ is the difference between inclusion and exclusion proportions. The $A$ (automatic retrieval) estimates were computed by dividing the exclusion proportions by $(1-C)$, and the means of these estimates are shown in Table 2. Because the mean inclusion and exclusion proportions for unstudied items were very similar, the baseline used to test for transfer was the average of the inclusion and exclusion proportions (as in Jacoby et al., 1993). The mean $C$ values were greater for deep than for shallow processing, for both visual and auditory conditions (sign tests, both $p$ s $<.001$ ). The mean $A$ values yielded a pattern similar to the one in the data for unrecognized items in the incidental test, with the visual-deep and auditory-deep conditions below baseline (sign tests, both $p s<.005$ ). In the visual-shallow condition, $A$ was significantly greater than baseline $[t(15)=2.33, p<.025]$, whereas in the auditoryshallow condition it was not $[t(15)=0.63, p>.25]$. The results from the shallow-processing conditions agree closely with prior reports showing within-modality, but not cross-modality, transfer of the $A$ parameter (Jacoby et al., 1993; Jacoby et al., in press). Taken in conjunction with our incidental-test results, they reproduce within a single experiment the apparent conflict between the results of the process-dissociation procedure and the incidentaltest results of Craik et al. (1994). They are, therefore, in accord with our hypothesis that the $A$ parameter does not reflect involuntary memory, both conscious and unconscious, but only involuntary unconscious memory.

The $A$ parameter showed a negative effect of depth of processing, so that $C$ and $A$ were inversely related as a function of that manipulation (as in Richardson-Klavehn \& Gardiner, 1995). This outcome contrasts with the results of Toth et al. (1994), who reported a depth-ofprocessing effect on $C$, but a null effect on $A$. In the process-dissociation framework, an inverse relationship between $C$ and $A$ is often treated as showing that the independence model for $C$ and $A$ is inapplicable under the particular conditions employed (see Jacoby et al., 1996; Jacoby et al., in press; Reingold \& Toth, 1996; Toth et al., 1995). The independence model is held to be inapplicable (1) when the test instructions do not induce participants to engage in voluntary retrieval of studied completions; or (2) when there is a floor effect on exclusion perfor- 
mance, which limits the boosting effect of the independence correction [i.e., the procedure of dividing exclusion performance by $(1-C)]$.

With respect to the first possibility, when voluntary retrieval is not recruited by the test instructions, the "signature" in the data is that inclusion-test performance resembles incidental-test performance (see, e.g., Jacoby et al., 1996). That possibility can therefore be ruled out here, because inclusion performance showed a strong depth-of-processing effect (indicating voluntary retrieval of studied completions) and because exclusion scores were derived from inclusion scores (ensuring that voluntary retrieval contributed equally to inclusion and exclusion scores). The first possibility is also clearly inconsistent with the current completion-time data. With respect to the second possibility, our deep-processing conditions can be regarded as showing a floor effect on exclusion performance, but our shallow-processing conditions cannot. There were no zero exclusion scores in the shallow conditions, and to confirm that the pattern in these conditions did not reflect a floor effect, we recomputed $A$ on the basis of mean inclusion and exclusion scores (as in Toth et al., 1994). The recomputed $A$ values for the visualshallow and auditory-shallow conditions were .28 and .22 , respectively, so that the pattern in these conditions did not change. Finally, we emphasize again that these results are consistent with - not divergent from-the results from prior comparisons of within- and cross-modality transfer of the automatic retrieval parameter (Jacoby et al., 1993; Jacoby et al., in press), despite our modification to the procedure.

\section{GENERAL DISCUSSION}

To summarize, our data show that memory that is involuntary, and unassociated with conscious awareness of memory, does not transfer across modalities. However, the current data, by replicating the results of Craik et al. (1994), also confirm that cross-modality priming should not be attributed to voluntary retrieval, as is done in the process-dissociation framework (see, e.g., Jacoby et al., 1993, Jacoby et al., in press) Instead, the results of the on-line recognition measure in our incidental test suggest that cross-modality priming is involuntary but associated with conscious awareness of memory. By equating conscious awareness of memory with voluntary retrieval, the process-dissociation model leads to the mistaken conclusion that cross-modality priming in incidental tests reflects contamination by voluntary retrieval.

Misattributing cross-modality priming in incidental tests to contamination by voluntary retrieval causes interesting and important theoretical possibilities to be overlooked. Perceptual priming reflects modalitydependent and modality-independent components that can be experimentally dissociated (e.g., Kirsner et al., 1989; Weldon, 1991, 1993). The within-modality component may reflect the operation of perceptual representation systems that operate in the absence both of voluntary retrieval and of memorial awareness (e.g., Tulving \& Schacter, 1990) The cross-modality component necessarily involves more abstract representations. It does not seem to depend on conceptual representations because priming in our incidental test was uninfluenced by depth of processing. Instead, the data further support hypotheses that attribute crossmodality priming to lexical representations (e.g., Weldon, 1991, 1993) or to representations for verbal responses that accumulate information across input modalities (e.g., Kirsner et al., 1989). The interesting additional implication of the current data is that the modality-independent component of perceptual priming, while involuntary, is more likely to be associated with memorial awareness than is the modality-specific component. Dividing attention at study eliminates cross-modality priming in an incidental test, an effect that has been interpreted as reflecting the elimination of voluntary contamination (Jacoby et al., in press). However, a more interesting interpretation of this effect is that dividing attention inhibits encoding of the modality-independent information that is responsible for involuntary cross-modality priming.

More generally, the current results illustrate the value of a framework that distinguishes retrieval volition (voluntary vs. involuntary) from memorial state of awareness (conscious vs. unconscious), accommodating involuntary conscious memory (e.g., Richardson-Klavehn et a! 1996). Critics of our approach have suggested that the phenomenon we refer to as involuntary conscious memory is in fact consistent with simple two-component models that equate voluntary retrieval with awareness of memory, and involuntary retrieval with the absence of such awareness (Reingold \& Toth, 1996; Toth et al., 1995). Their argument is that "involuntary conscious memory" occurs when involuntary unconscious retrieval and voluntary conscious retrieval co-occur on a given test trial, but there is a brief time lag between the former and the latter (Reingold \& Toth, 1996). This suggestion, however, cannot accommodate our data, because it still predicts that whenever there is memorial awareness, there is voluntary retrieval. For example, deep processing at encoding typically led to memorial awareness in our incidental test, as indicated by our on-line recognition measure. One would then have to predict that priming in that test should be contaminated by voluntary retrieval of studied items. In contrast, there was no evidence at all that priming reflected voluntary retrieval. In sum, the results presented here and elsewhere (Richardson-Klavehn \& Gardiner, 1995) demonstrate dissociations between retrieval volition and awareness of memory that cannot be accommodated by any simple two-component model.

\section{REFERENCES}

Challis, B. H., \& Brodbeck, D. R. (1992). Level of processing affects priming in word fragment completion. Journal of Experimental Psychology: Learning, Memory, \& Cognition, 18, 595-607.

Craik, F. I. M., Moscovitch, M., \& McDowd, J. M. (1994). Contributions of surface and semantic information to performance on implicit and explicit memory tasks. Journal of Experimental Psychology: Learning, Memory, \& Cognition, 20, 864-875

Ebbinghaus, H. (1964). Memory: A contribution to experimental psychology (H. A. Ruger \& C. E. Bussenius, Trans.). New York: Dover (Original work published 1885)

JACOBY, L. L. (1991). A process dissociation framework: Separating automatic from intentional uses of memory. Journal of Memory \& Language, 30, 513-541.

JACOBY, L. L., BEGG, I. M., \& ToтH, J. P. (1996). In defense of functional independence: Violations of assumptions underlying the processdissociation procedure? Manuscript submitted for publication.

Jacoby, L. L., Toth, J. P., \& Yonelinas, A. P. (1993). Separating conscious and unconscious influences of memory: Measuring recollection. Journal of Experimental Psychology: General, 122, 139-154.

JACOBY, L. L., Yonelinas, A. P., \& Jennings, J. (in press). The relation between conscious and unconscious (automatic) influences: A declaration of independence. In J. D. Cohen \& J. W. Schooler (Eds.). Scientific approaches to the question of consciousness. Hillsdale, NJ: Erlbaum.

Kirsner, K., Dunn, J. C., \& Standen, P. (1989). Domain-specific resources in word recognition. In $\mathrm{S}$. Lewandowsky, J. C. Dunn, \& K. Kirsner (Eds.), Implicit memory: Theoretical issues (pp. 99-122). Hillsdale, NJ: Erlbaum.

Rajaram, S., \& Roediger, H. L., III (1993). Direct comparison of four implicit memory tests. Journal of Experimental Psychology: Learning, Memory, \& Cognition, 19, 765-776.

Reingold, E. M., \& TOTH, J. P. (1996). Process dissociations versus task dissociations: A controversy in progress. In G. Underwood (Ed.), Implicit cognition (pp. 159-202). Oxford: Oxford University Press.

Richardson-KLAVEHN, A., \& BJoRK, R, A. (1988). Measures of memory. Annual Review of Psychology, 39, 475-543.

RiChardson-Klavehin, A., \& Gardiner, J. M. (1995). Retrieval volition and memorial awareness in stem completion: An empirical analysis. Psychological Research, 57, 166-178. 
Richardson-Klavehn, A., Gardiner, J. M., \& JAVA, R. I. (1994). Involuntary conscious memory and the method of opposition. Memory, 2, 1-29.

Richardson-Klavehn, A., Gardiner, J. M., \& JaVa, R. I. (1996). Memory: Task dissociations, process dissociations and dissociations of consciousness. In G. Underwood (Ed.), Implicit cognition (pp. 85158). Oxford: Oxford University Press.

Richardson-Klavehn, A., Lee, M. G., Joubran, R., \& BJork, R. A. (1994). Intention and awareness in perceptual identification priming. Memory \& Cognition, 22, 293-312.

ROEDIGER, H. L., III, \& MCDERMOTT, K. B. (1993). Implicit memory in normal human subjects. In F. Boller \& J. Grafman (Eds.), Handbook of neuropsychology (Vol. 8, pp. 63-131). Amsterdam: Elsevier.

Roediger, H. L., III, Weldon, M. S., Stadler, M. L., \& Riegler, G. L. (1992). Direct comparison of two implicit memory tests: Word fragment and word stem completion. Journal of Experimental Psychology: Learning, Memory, \& Cognition, 18, 1251-1269.

SCHACTER, D. L. (1987). Implicit memory: History and current status. Journal of Experimental Psychology; Learning, Memory, \& Cognition, 13, 501-518.

SCHACTER, D. L., Bowers, J., \& BoOKER, J. (1989). Intention, awareness, and implicit memory: The retrieval intentionality criterion. In S. Lewandowsky, J. C. Dunn, \& K. Kirsner (Eds.), Implicit memory: Theoretical issues (pp. 47-65). Hillsdale, NJ: Erlbaum.

Schacter, D. L., Reiman, E., Uecker, A., Polster, M. R., Yun, L. S. \& COOPER, L. A. (1995). Brain regions associated with retrieval of structurally coherent visual information. Nature, 376, 587-590.

ToTH, J. P., \& REINGOLD, E. M. (1996). Beyond perception: Conceptual contributions to unconscious influences of memory. In G. Underwood (Ed.), Implicit cognition (pp. 41-84). Oxford: Oxford University Press.

Toth, J. P., Reingold, E. M., \& JACOBY, L. L. (1994). Toward a redefinition of implicit memory: Process dissociation following elaborative processing and self-generation. Journal of Experimental Psychology: Learning, Memory, \& Cognition, 20, 290-303.
Toth, J. P., Reingold, E. M., \& JACOBY, L. L. (1995). A response to Graf and Komatsu's critique of the process-dissociation procedure: When is caution necessary? European Journal of Cognitive Psychology, 7, 113-130.

Tulving, E., \& Schacter, D. L. (1990). Priming and human memory systems. Science, 247, 301-305.

WELDON, M. S. (1991). Mechanisms underlying priming on perceptual tests. Journal of Experimental Psychology: Learning, Memory, \& Cognition, 17, 526-541.

WeLDON, M. S. (1993). The time course of perceptual and conceptual contributions to word fragment completion priming. Journal of Experimental Psychology: Learning, Memory, \& Cognition, 19, 1010-1023.

\section{NOTE}

1. Time to complete the test was not recorded for 1 participant in the intentional test owing to experimenter error. The incidental-test group wrote fewer additional words in total $(M=11.1)$ than the intentionaltest group $(M=16.7)$, so that the difference in time between the groups might reflect the time taken just to write the additional words down (about $2 \mathrm{sec}$ per word, according to a conservative empirical estimate obtained by Richardson-Klavehn and Gardiner, 1995). Prior to computing the time per item, therefore, 2 sec per additional word written were deducted from each participant's total time. The results of the posttest interview are consistent with the time difference between groups. None of the 16 incidental-test participants stated that they had tried to retrieve studied words. By contrast, 14 of the 16 intentional-test participants stated that they had tried to retrieve studied words, and only 2 stated that they had given the first word that came to mind. All information presented here relates to participants who successfully completed the experiment (and not to the 3 participants who were replaced because they failed to follow test instructions).

(Manuscript received March 14, 1995; revision accepted for publication November 9, 1995.) 